\title{
Optimized methods for Legionella pneumophila release from its Acanthamoeba hosts
}

\author{
Elisabeth Dietersdorfer ${ }^{1 \dagger}$, Sílvia Cervero-Aragó ${ }^{2,3^{*}+}$, Regina Sommer ${ }^{2,3}$, Alexander K. Kirschner ${ }^{2,3}$
} and Julia Walochnik'

\begin{abstract}
Background: Free-living amoebae (FLA) and particularly acanthamoebae serve as vehicles and hosts for Legionella pneumophila, among other pathogenic microorganisms. Within the amoebae, L. pneumophila activates a complex regulatory pathway that enables the bacteria to resist amoebal digestion and to replicate. Moreover, the amoebae provide the bacteria protection against harsh environmental conditions and disinfectants commonly used in engineered water systems. To study this ecological relationship, co-culture and infection models have been used. However, there is a lack of data regarding the effectiveness of the different methods used to release intracellular bacteria from their amoebal hosts. The aim of this study was to evaluate the impact of the methods used to release intracellular L. pneumophila cells on the culturability of the bacteria. Furthermore, the standard method ISO 11731:1998 for the recovery and enumeration of Legionella from water samples was evaluated for its suitability to quantify intracellular bacteria.
\end{abstract}

Results: The effectiveness of the eight release treatments applied to L. pneumophila and Acanthamoeba strains in a free-living state varied between bacterial strains. Moreover, the current study provides numerical data on the state of co-culture suspensions at different time points. The release treatments enhanced survival of both microorganisms in co-cultures of L. pneumophila and Acanthamoeba. Passage through a needle $(21 \mathrm{G}, 27 \mathrm{G})$ and centrifugation at $10,000 \times g$ showed the highest bacterial counts when releasing the bacteria from the intracellular state. Regarding the ISO 11731:1998 method, one of the tested strains showed no differences between the recovery rates of associated and free-living L. pneumophila. However, a reduced bacterial recovery rate was observed for the second L. pneumophila strain used, and this difference is likely linked to the survival of the amoebae.

Conclusions: Mechanical release treatments were the most effective methods for providing bacterial release without the use of chemicals that could compromise further study of the intracellular bacteria. The current results demonstrated that the recovery of L. pneumophila from water systems may be underestimated if protozoal membranes are not disrupted.

Keywords: L. pneumophila, Endosymbiont, Acanthamoeba, Release

\footnotetext{
* Correspondence: silvia.cerveroarago@meduniwien.ac.at

${ }^{\dagger}$ Equal contributors

${ }^{2}$ Institute for Hygiene and Applied Immunology, Water Hygiene, Medical

University of Vienna, Kinderspitalgasse 15, A-1090 Vienna, Austria

${ }^{3}$ Interuniversity Cooperation Centre for Water \& Health, Vienna, Austria

Full list of author information is available at the end of the article
} 


\section{Background}

Free-living amoebae (FLA), particularly acanthamoebae, have recently gained scientific attention not only because of their intrinsic pathogenicity [1-3] but also because they serve as vehicles and hosts for a wide range of pathogenic microorganisms, such as Coxiella burnetti, Chlamydophila pneumoniae, Mycobacterium spp., Pseudomonas aeruginosa, Vibrio cholera and Legionella pneumophila [4-7].

Among these bacteria, the relationship between Acanthamoeba and L. pneumophila is one of the most studied due to the associated health risk. Legionellae are Gramnegative bacteria and common inhabitants of aquatic environments [8, 9]. Engineered habitats such as drinking water systems, hot water systems and cooling towers [10] may provide optimal conditions for the replication of these bacteria associated with protozoa. Legionellae are transmitted to humans via aerosols produced from these contaminated water sources and cause a severe lung infection called Legionnaires' disease or a milder influenza-like form known as Pontiac Fever [11].

A wide range of disinfection techniques has been applied to control and prevent Legionella proliferation in drinking water systems [12]; however, cases of legionellosis may still occur. The protection from harsh environmental conditions and disinfectants that amoebae provide to intracellular bacteria has been studied in detail [13-16]. Furthermore, the molecular regulators that L. pneumophila uses at the transcriptional and (or) post-transcriptional level to control the expression of virulence traits and fitness factors to adapt to different intracellular (amoebae and macrophages) or extracellular environments have been revealed [17]. Additionally, various L. pneumophila-Acanthamoeba co-culture and infection models using chemical as well as mechanical methods to release the intracellular bacteria from their amoebal hosts have been described [18-26]. However, there is a lack of data regarding the different treatments and their effects on L. pneumophila.

The aim of this work was to establish a standard method for the release of intracellular L. pneumophila by testing and comparing several published and nonpublished protocols. Moreover, the effectiveness of the standard method commonly used for the isolation and quantification of Legionella spp. from water samples (ISO 11731:1998 [27]) was evaluated for its suitability for intracellular bacteria. Our results indicate that this method is prone to underestimating the number of surviving bacterial cells because L. pneumophila replicating vesicles within amoebae will grow as singles colonies on agar plates if the protozoal membranes are not disrupted. The current study provides optimised methods for the study of Acanthamoeba-Legionella interactions and of intracellular L. pneumophila from environmental samples.

\section{Results and discussion}

There is a lack of information in the literature regarding the effectiveness of the methods used to release intracellular bacteria from amoeba hosts. In the current study, we assessed different approaches of release methods to determine the most appropriate method for each purpose.

\section{Effect of release treatments on pure cultures}

To evaluate potential treatments for the release of intracellular bacteria, suspensions of two different $L$. pneumophila strains and two different Acanthamoeba strains were initially tested in a free-living state. Optimal treatments would result in a minimum log reduction of bacterial culturability but a high log reduction in amoebal culturability.

Among the eight treatments tested, mechanical treatments such as the passage through 21G and 27G needles, freezing-thawing cycles and treatment with liquid $\mathrm{N}_{2}$ resulted in a loss of approximately 1 log of cultivable L. pneumophila cells (Fig. 1). In contrast, chemical treatments using SDS and Triton ${ }^{\mathrm{TM}} \mathrm{X}-100$ reduced L. pneumophila culturability by 3-4.5 logs. That fact shows that the membrane composition of L. pneumophila particularly rich in branched fatty acids [28] is very sensitive to the effect of such detergents. Moreover, these results are in agreement with Moffat et al. [22], who noted that the use of Triton ${ }^{\mathrm{Tm}} \mathrm{X}-100$ could damage L. pneumophila cells even at low concentrations. Interestingly, significant differences were observed between the two L. pneumophila strains tested. The L. pneumophila Paris strain was significantly more susceptible to treatment with Triton $^{\mathrm{TM}} \mathrm{X}-100(P<0.001)$, freezing-thawing $(P<0.01)$, centrifugation $(P<0.001)$ and $\mathrm{KCl}(P<0.001)$ than the L. pneumophila Olda strain, particularly concerning the last two treatments. These results are in agreement with other authors who have already reported differences between L. pneumophila strains of the same serogroup towards drinking water disinfection methods [29]. Thus, preliminary tests should be performed on each L. pneumophila strain before choosing a release method.

Regarding the amoebae, the chemical treatment with SDS and the mechanical treatments based on freezingthawing and liquid $\mathrm{N}_{2}$ were the most effective; and reduced their culturability by $2-3$ logs (Fig. 2). Although the resistance to a wide range of temperatures of some Acanthamoeba strains in a cystic live stage is well known $[13,30]$, the trophozoites of the two strains used were very sensitive to the thermal treatments applied. In contrast, the other release treatments reduced amoebal culturability by less than $1 \mathrm{log}$. No significant differences were observed between the two Acanthamoeba strains tested for any of the treatments applied $(P>0.05)$. 


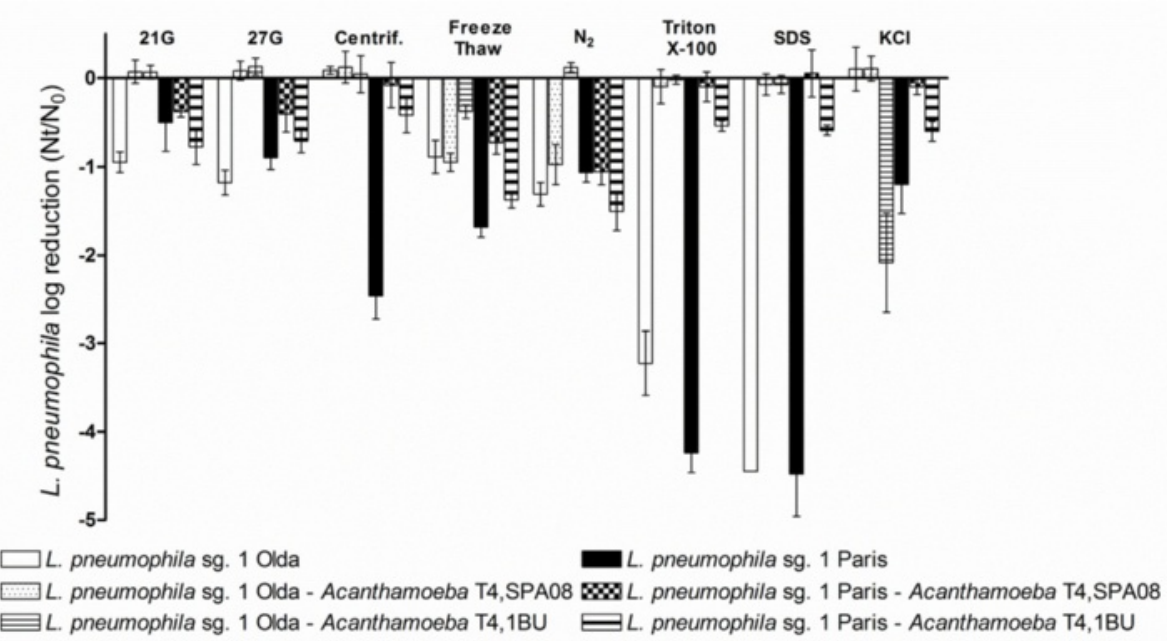

Fig. 1 Effect of the eight release treatment methods applied to the free-living L. pneumophila strains and to co-cultures of the two L. pneumophila strains and the two Acanthamoeba strains. L. pneumophila inactivation was determined using viable counts on supplemented BCYE agar medium. Data are presented as the means \pm SD (columns and error bars)

Because none of the treatments tested fulfilled the requirements to be considered optimal and due to the high variability between $L$. pneumophila strains, all eight treatments were tested also on the co-cultures of amoebae and bacteria.

\section{Intracellular growth of $L$. pneumophila within Acanthamoeba}

The intracellular growth of two L. pneumophila strains within two different Acanthamoeba strains was monitored using the MONOFLUO ${ }^{\mathrm{mm}}$ Legionella pneumophila
IFA Test Kit (Fig. 3). The commercial kit consisted of FITC-labelled monoclonal antibodies that bind to the major outer membrane protein (MOMP) of L. pneumophila (in green) and a counterstain that labelled amoebal membranes (in red). This method resulted in a very useful, quick and easy manner of monitoring the bacterial state in co-culture suspensions. For the L. pneumophila Paris strain co-cultures, pictures were taken only for $24 \mathrm{~h}$ because after that time almost all of the bacterial cells were again in an extracellular state after bursting their amoebal hosts. All suspensions were

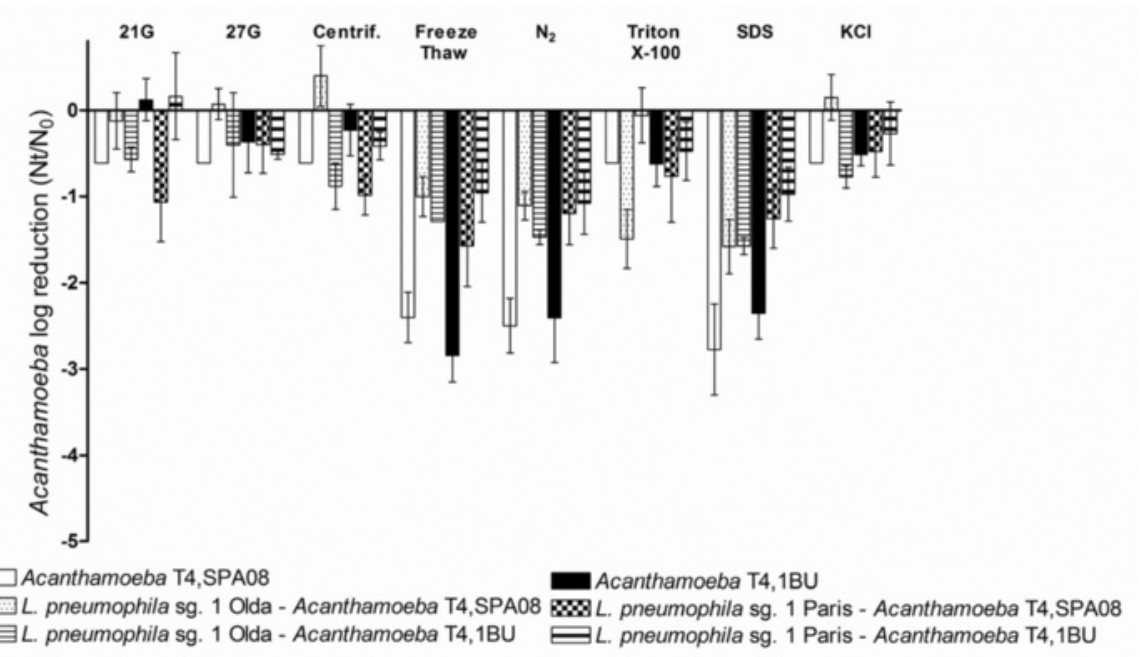

Fig. 2 Effect of the eight release treatments methods applied to the free-living Acanthamoeba strains and to the co-cultures of the two L. pneumophila strains and the two Acanthamoeba strains. Amoeba inactivation was determined using the MPN method on NNA plates. Data are presented as the means \pm SD (columns and error bars) 


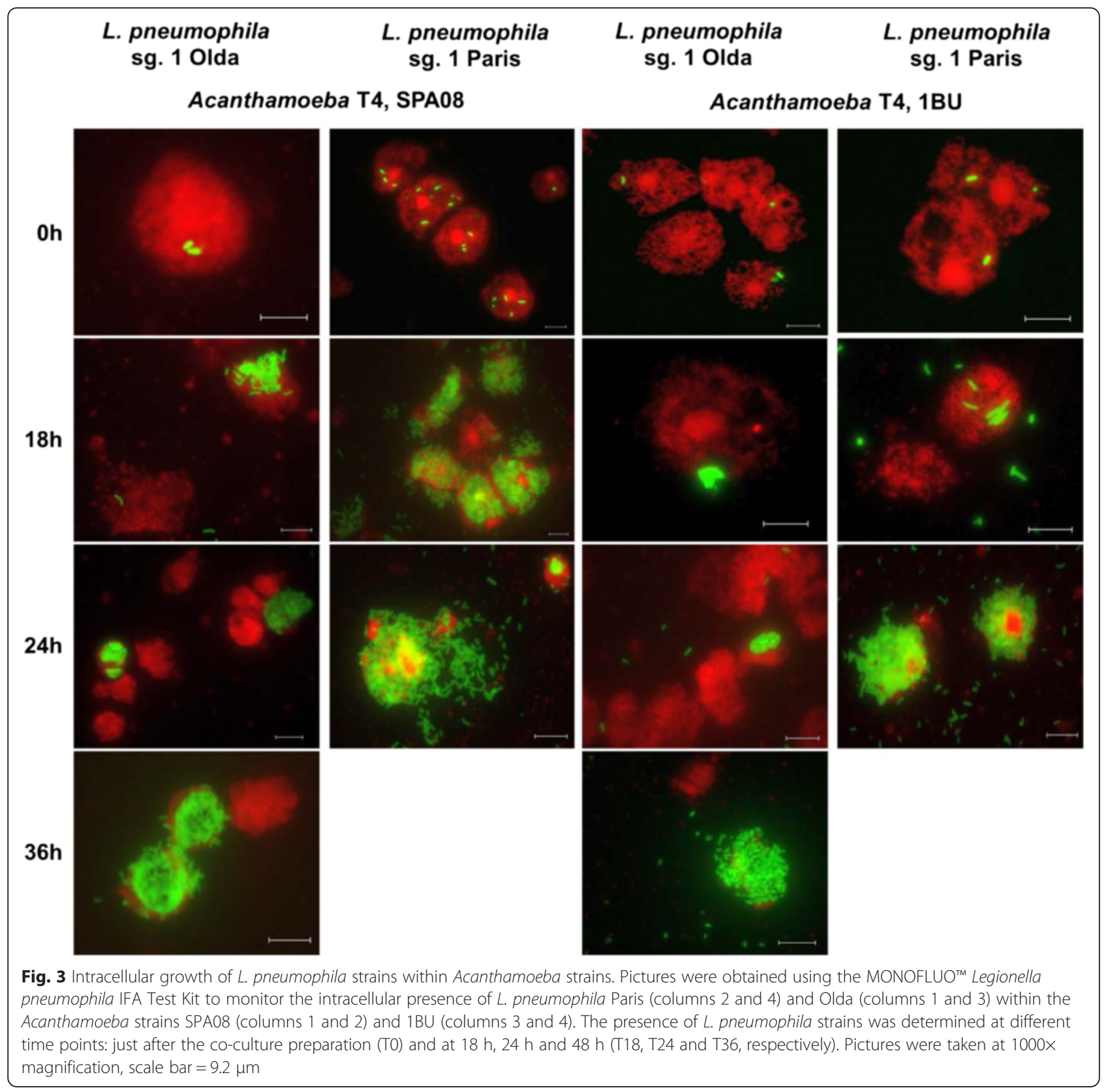

analysed at different time points after co-culture and the number of infected and non-infected amoeba was assessed as well as the number of legionellae within every amoeba (Fig. 4). The state of the co-cultures in terms of the number of intracellular bacteria per amoeba was defined by using 4 different categories: 1 . non-infected, 2. low-infected $(<10 \mathrm{~L}$. pneumophilal amoeba, early stage of infection), 3. medium-infected (quantifiable replicating L. pneumophila cells), 4. highinfected (amoebae full of L. pneumophila cells, no cytosol left). According to the data obtained we determined the infectivity rate of each L. pneumophila strain, grouping categories 2, 3 and 4 versus category 1 , and the time necessary to replicate in the amoeba host (Fig. 4). A similar approach to describe bacterial and amoebal interactions has been previously used [31].

The ability to replicate within amoeba hosts has been described by several authors as a strain-dependent event $[29,32]$. The current study revealed that the extent of intracellular growth of L. pneumophila within Acanthamoeba indeed varies depending on both the bacterial and the amoebal strains used (Fig. 4). For example, amoebae of the category 4 with no cytosol 

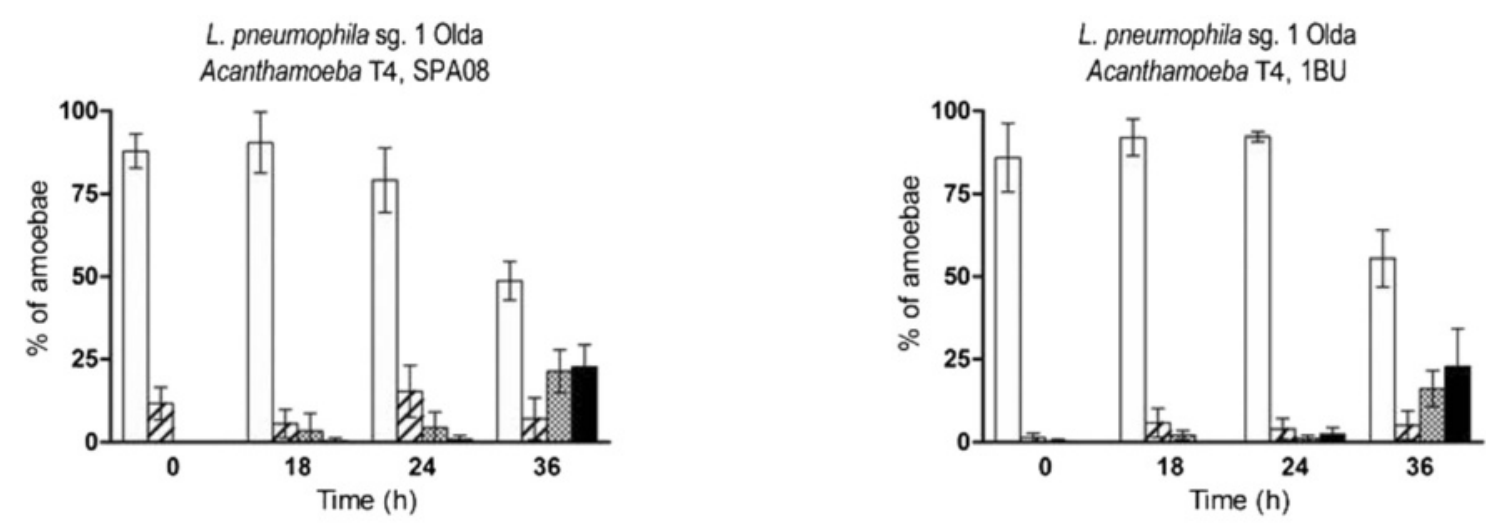

Not infected ZZL Low infected Medium infected Migh infected
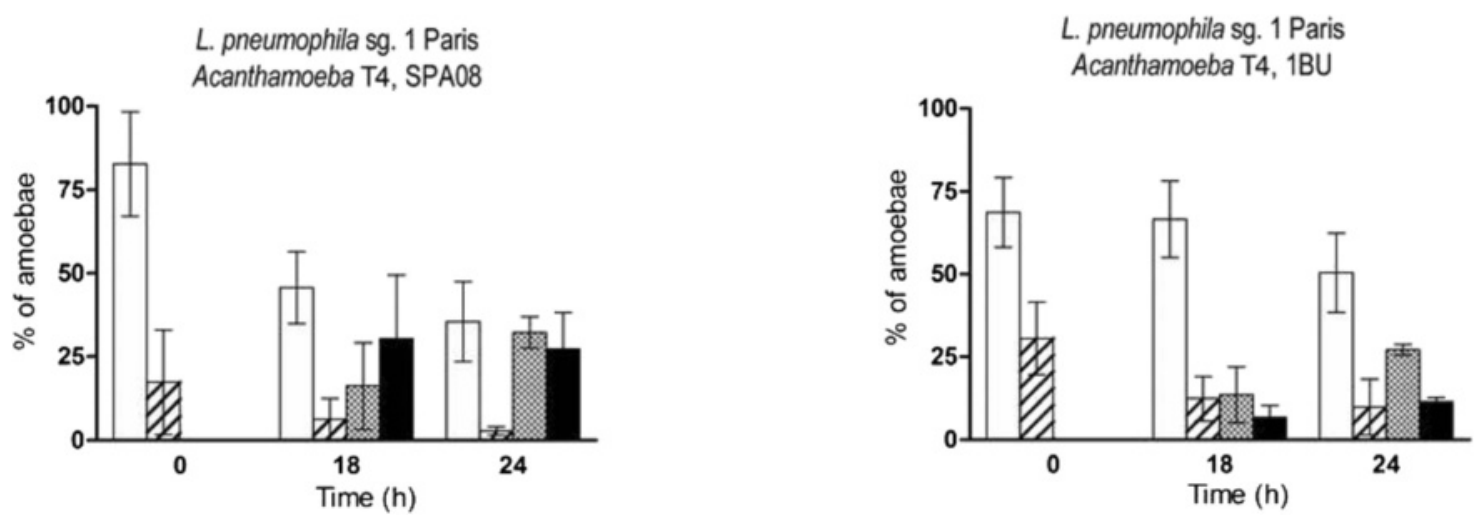

Fig. 4 Efficiency of intracellular growth of L. pneumophila Paris and Olda within Acanthamoeba SPA08 and 1BU at different time points. Bacterial and amoebae cells were stained using the MONOFLUO ${ }^{\text {TM }}$ Legionella pneumophila IFA Test Kit and counted using an epifluorescent microscope. Amoebae were divided in four different categories to describe their state: not-infected, low-infected $(<10$ L. pneumophila/amoeba, early stage of infection), medium-infected (quantifiable replicating L. pneumophila cells) and high-infected (non-quantifiable, amoebae full of L. pneumophila cells with no cytosol left). Data are presented as the percentage \pm SD (columns and error bars)

left hosted around 40-50 bacterial cells whereas other amoebae hosted more than 100. The L. pneumophila Paris strain showed a higher virulence than the $L$. pneumophila Olda strain. This fact was represented first, by a higher infectivity rate, the L. pneumophila Paris strain infected a higher number of amoebal cells in $24 \mathrm{~h}, 62 \pm 17 \%$ in co-culture with the Acanthamoeba SPA08 strain and $49 \pm 11 \%$ in co-culture with the Acanthamoeba $1 \mathrm{BU}$ strain whereas, the L. pneumophila Olda strain infected $21 \pm 13 \%$ of the Acanthamoeba SPA08 strain and $8 \pm 6 \%$ of the Acanthamoeba $1 \mathrm{BU}$ strain at the same time point (Fig. 4). Second, L. pneumophila Paris strain was faster in replicating within the two Acanthamoeba hosts since after $18 \mathrm{~h}$ of co-culture $30 \pm 19 \%$ of Acanthamoeba SPA08 cells and $7 \pm 3 \%$ of Acanthamoeba 1BU cells were high-infected (category 4) (Fig. 4). However, it was necessary to prolong the co-cultures up to $36 \mathrm{~h}$ to observe more than $7 \%$ of high-infected amoeba cells for the L. pneumophila Olda strain (Fig. 4).

The time point used in the release experiments was chosen according to the maximum number of amoeba infected and the maximum number of bacterial cell observed within them, category 3 and 4 (Fig. 4). Thus, treatments were applied after $24 \mathrm{~h}$ for the L. pneumophila Paris strain co-cultures and $36 \mathrm{~h}$ for the L. pneumophila Olda strain co-cultures.

\section{Effect of release treatments on co-cultures}

To determine the best way to recover intracellular $L$. pneumophila, the eight release treatments were applied to the four co-cultures established.

The effectiveness of each treatment in releasing the intracellular bacteria was shown to depend on the respective strains (Fig. 1). Overall, the highest effectiveness, represented by the lowest loss of culturability, was 
observed in the L. pneumophila Olda co-cultures. The results of treatments such as passage through $21 \mathrm{G}$ and 27G needles and chemical treatments with SDS and Triton $^{\text {TM }}$ X-100 varied from $1 \log$ to 4 log reduction in free-living bacteria suspensions to less than $0.5 \log$ reduction or no reduction in co-culture for the L. pneumophila Olda strain. This fact confirms that most of Acanthamoeba membranes were disrupted by the release methods and beyond that Acanthamoeba cells play a protective role for $L$. pneumophila cells, as previously reported [14, 29]. Regarding the $\mathrm{KCl}$ treatment $(P<0.001)$ L. pneumophila Olda was divergently reduced depending on the Acanthamoeba co-culture. In this case, it was not clear whether the release treatment was not strong enough to disrupt amoebal membranes or in contrast with the studies by Berk et al. [18] and Holden et al. [33] the amoebal protection was not sufficient. Moreover, Barker et al. [28] reported changes in the membrane composition of intra-amoebic L. pneumophila, which could also explain some of the differences observed between intra- and extracellular states. There was a similar trend for the L. pneumophila Paris strain, although a higher variability between the treatments was observed (Fig. 1). Methods such as centrifugation, Triton $^{\text {Tx }} \mathrm{X}-100$, SDS, freezing-thawing and $\mathrm{KCl}$ treatments resulted in a 2 to $4 \log$ reduction of the culturability for $L$. pneumophila Paris suspensions but in less than $1 \log$ reduction in co-culture with Acanthamoeba. As observed for the L. pneumophila Olda strain, the release treatment with liquid $\mathrm{N}_{2}$ was not sufficient to disrupt Acanthamoeba 1BU membranes. Comparing the two co-cultures of the L. pneumophila Paris strain, the highest loss of culturability was linked to the Acanthamoeba $1 \mathrm{BU}$ strain. Among the treatments applied, centrifugation and Triton $^{\mathrm{nm}} \mathrm{X}-100$ treatments were the most effective for recovering cultivable cells. Interestingly, despite the harmful effect of Triton ${ }^{\mathrm{TM}} \mathrm{X}-100$ on free-living cells, this detergent has been used in many co-culture studies [13, 23, 34].

Regarding amoebal counts, it was shown that the effects of the release treatments tested were rather similar between the two Acanthamoeba strains (Fig. 2). The most effective methods, reducing amoeba counts by approximately 1.5 logs, were the treatments with SDS, freezing-thawing and liquid $\mathrm{N}_{2}$. However, these treatments also considerably reduced the culturability of $L$. pneumophila. Moreover, co-culture with $L$. pneumophila cells also reduced Acanthamoeba susceptibility to the treatments applied. For Acanthamoeba strain SPA08, the association with $L$. pneumophila Olda reduced the effect of centrifugation and liquid $\mathrm{N}_{2}$ whereas the association with L. pneumophila Paris reduced the effect of SDS and liquid $\mathrm{N}_{2}$. Moreover, for Acanthamoeba 1BU, the association with $L$. pneumophila Olda reduced the effect of freezing-thawing whereas the association with $L$. pneumophila Paris reduced the effect of treatment with SDS. Thus, co-culture between L. pneumophila and Acanthamoeba strains provides a reciprocal resistance to the applied treatments, as has been previously observed [34]. To understand the mechanisms of this reciprocal protection, further experiments are needed. In summary, the current study showed that mechanical release treatments such as passage through $21 \mathrm{G}$ and $27 \mathrm{G}$ needles as well as centrifugation can be considered optimal for the release of L. pneumophila from Acanthamoeba strains without the use of chemicals that could interfere with further experiments.

\section{Evaluation of the ISO 11731:1998 method in co-cultures} Suspensions of L. pneumophila and Acanthamoeba microorganisms in a free-living state and associated in co-culture were processed following the ISO 11731:1998 method for the detection and enumeration of Legionella [27].

Despite the differences in the response to the release treatments between the two L. pneumophila strains as pure cultures, no significant differences $(P>0.05)$ were found in their recovery rates following the ISO method (Fig. 5). Direct plating and acid treatment resulted in a $\log$ reduction of culturability lower than $0.5 \operatorname{logs}$ for the L. pneumophila strains, whereas heat treatment reduced culturability by almost $1 \mathrm{log}$. No significant differences $(P>0.05)$ were found in the recovery of non-associated L. pneumophila Olda and L. pneumophila Olda associated with the Acanthamoeba SPA08 strain for any of the methods analysed. However, the association with the Acanthamoeba $1 \mathrm{BU}$ strain, despite what was observed for most of the release treatments applied, significantly increased $(P<0.001)$ the susceptibility of both $L$. pneumophila strains to the heat treatment. The recovery of the L. pneumophila Paris strain notably varied depending on the bacterial state. Although no significant differences were found between the two co-cultures with Acanthamoeba strains, the recovery of the bacteria significantly decreased when compared with the free-living state for the direct plating $(P<0.001)$, heat treatment $(P<$ $0.001)$ and acid treatment $(P<0.001)$ methods. This result could indicate that in the case of the L. pneumophila Paris strain, the standard method might not be harsh enough to disrupt the amoebal membranes. Thus, the replicating L. pneumophila vesicles from inside the amoebae grew as single colonies on the agar plates, resulting in an underestimation of the real number of bacterial cells. The analysis of amoebal recovery with the ISO method showed no significant differences $(P>0.05)$ between the two Acanthamoeba strains without bacteria (Fig. 5). The direct plating, heat treatment and acid treatment reduced amoeba culturability by approximately $1 \mathrm{log}$, $3.5 \operatorname{logs}$ and 1.5 logs, respectively. The comparison between amoebae that were associated and those non- 

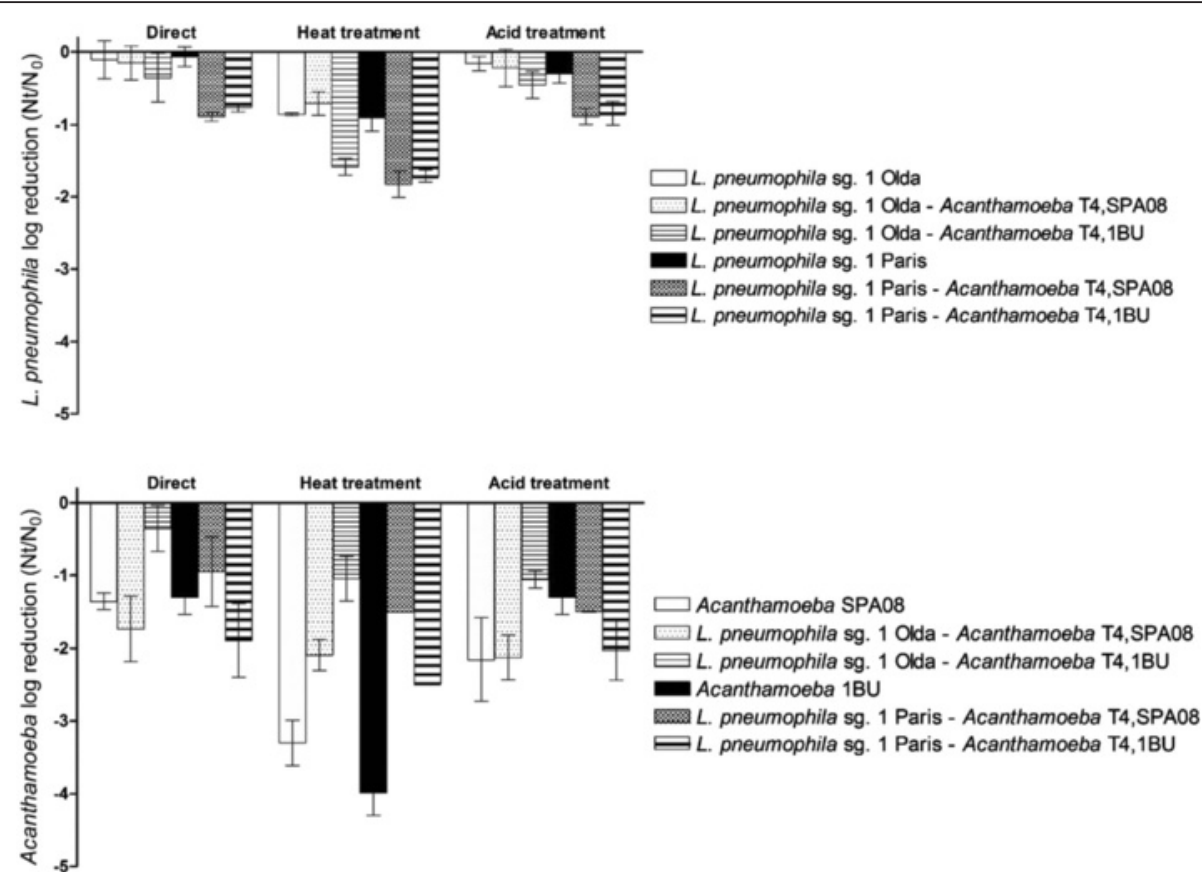

Fig. 5 Effectiveness of the ISO 11731:1998 method in the recovery of L. pneumophila Paris and Olda and Acanthamoeba SPA08 and 1BU in a free-living state and in co-culture. Bacterial inactivation was determined using viable counts on supplemented BCYE agar medium, and amoebal inactivation was determined using the MPN method. Data are presented as the means \pm SD (columns and error bars)

associated with L. pneumophila strains revealed that the association of Acanthamoeba SPA08 with the bacteria significantly reduced its susceptibility to the heat treatment $(P<0.001)$. In the case of Acanthamoeba 1BU, a similar behaviour was observed for the direct plating $(P<$ $0.001)$ as well as for the heat treatment $(P<0.001)$. Thus, co-culture with bacteria enhanced amoebal survival. This phenomenon could explain the low recovery rates of the L. pneumophila Paris strain. Thus, depending on the L. pneumophila strains and their state, associated with amoebae or as free cells, their numbers could be significantly underestimated when using the ISO 11371:1998 method.

\section{Conclusions}

In the current work, several release methods for the study of intracellular bacteria were assessed. Co-culture models were established using L. pneumophila and its amoebal host Acanthamoeba. Using an immunofluorescence assay specific for Legionella pneumophila proved to be extremely useful to monitor the intracellular state of the bacteria and to determine strain virulence. The investigation of the methods for intracellular L. pneumophila release from within Acanthamoeba host cells showed that mechanical release treatments, namely three passages through a $21 \mathrm{G}$ or $27 \mathrm{G}$ needle or centrifugation at $10,000 \times \mathrm{g}$ for $10 \mathrm{~min}$, were the most effective treatments, showing the highest recovery rates without risk of interference by chemical residuals. When applying the ISO 11371:1998 method for the recovery of environmental $L$. pneumophila from water samples, the number of intracellular bacteria may be underestimated if the amoebal membranes are not destroyed during the detection procedure. The co-analysis of amoebal hosts such as acanthamoebae in positive samples for $L$. pneumophila could help to obtain more accurate results. To avoid the underestimation of Legionella spp. concentration in water samples, the inclusion of a mechanical pretreatment step (e.g., needle passage) in the ISO 11371:1998 enumeration method might be considered.

\section{Methods}

\section{L. pneumophila strains}

Release studies were conducted with 2 Legionella pneumophila SG1 strains. The type strain OLDA (ATCC43109) was provided by the AGES (Austrian Agency for Food and Health Security) strain collection, and the environmental isolate PARIS [35] was kindly donated by Y. Héchard, University of Poitiers. Strains were embedded in cryobeads (Roti ${ }^{\circ}$-Store Cryovials, Roth, Germany) and stored at $-80{ }^{\circ} \mathrm{C}$.

\section{Acanthamoeba strains}

Experiments with amoebae were performed using 2 different Acanthamoeba genotype T4 strains 1BU (ATCC PRA-105) and SPA08 [36], both of which were isolated 
from keratitis patients. The strains were cultivated on non-nutrient-agar (NNA) plates previously seeded with heat-killed E. coli at room temperature (RT). From the NNA plates, axenic cultures were obtained according to the method described elsewhere [37]. Briefly, mature cysts were harvested from NNA plate cultures and incubated in $3 \% \mathrm{HCl}$ overnight to eliminate possible remaining bacteria. Suspensions were washed with Ringer solution 1/40 by centrifugation at $800 \mathrm{~g}$ for $15 \mathrm{~min}$ and transferred to $10 \mathrm{~mL}$ of PYG (proteose-peptone-yeast extract-glucose) medium (ATCC 712) media in $25 \mathrm{~cm}^{3}$ tissue culture flasks. Trophozoite cultures were maintained axenically by subculturing them in PYG in $25 \mathrm{~cm}^{3}$ culture flasks.

\section{Preparation of test suspensions and quantification of culturability after treatments \\ L. pneumophila suspensions}

L. pneumophila strains were cultured on supplemented GVPC BCYE (buffered charcoal-yeast extract) agar (Biomerieux) at $37{ }^{\circ} \mathrm{C}$ for $72 \mathrm{~h}$. Suspensions were prepared as described in Cervero-Aragó et al. [15]. Briefly, cells were harvested from the agar plates, resuspended in Ringer solution $1 / 40$ and homogenized using a vortex mixer. The cell concentrations of the respective suspensions were adjusted by measuring the absorbance at $450 \mathrm{~nm}$, using a photometer (Eppendorf Biophotometer). The concentrations of the tested Legionella suspensions were approximately $5 \times 10^{5} \mathrm{cfu} / \mathrm{mL}$. The treatments were applied as explained in Table 1.

\section{L. pneumophila quantification after treatments}

After each of the release treatments, ten-fold serial dilutions were prepared in Ringer solution 1/40 solution and transferred to supplemented BCYE plates for the enumeration of Legionella colony-forming units. The plates were incubated at $37{ }^{\circ} \mathrm{C}$ for up to 10 days and checked every 3-4 days.

\section{Acanthamoeba suspensions}

After the trophozoites had grown to confluence for 2-3 days at $30{ }^{\circ} \mathrm{C}$ in PYG medium, they were recovered from the tissue culture flasks with a soft shake and adjusted to a final concentration of $5 \times 10^{5}$ amoeba cells $/ \mathrm{mL}$ using a haemocytometer.

\section{Acanthamoeba quantification after treatments}

The concentration of viable of Acanthamoeba cells was quantified following the most probable number (MPN) method described elsewhere [38]. After the treatments ten-fold serial dilutions were prepared in Ringer solution $1 / 40$ and transferred to NNA plates previously seeded with five spots of $20 \mu \mathrm{l}$ of a fresh culture of E.coli Ten microliters of the diluted sample were added to these spots and plates were incubated at $30{ }^{\circ} \mathrm{C}$ for 8 days and checked every 2 days using an inverted microscope (Olympus CK2). The absence/presence of trophozoites in a dilution spot was considered negative/positive. The MPN values were obtained from MPN tables [39].

\section{Acanthamoeba-L. pneumophila co-cultures}

Acanthamoeba trophozoites were harvested by gently shaking the culture flasks to detach the cells and then transferring them to a $50 \mathrm{~mL}$ tube. Suspensions were counted as described above, and $5 \times 10^{5}$ trophozoites were transferred to a 12-well cell culture plate. Trophozoites were incubated at $30{ }^{\circ} \mathrm{C}$ for $1 \mathrm{~h}$ to enable their adherence to the plate wells. After that, the PYG medium was replaced by a dilution 1:10 of PYG medium in Page's amoeba saline (PAS) (ATCC1323, [40]), and L. pneumophila suspensions were added at a ratio of 100 legionellae per amoeba. To enhance the interaction between

Table 1 Description of the release treatments methods applied to L. pneumophila strains and Acanthamoeba strains in a free-living state or in co-culture

\begin{tabular}{|c|c|c|}
\hline Release treatments methods & Description & Reference \\
\hline \multicolumn{3}{|l|}{ Mechanical } \\
\hline 21 gauge needle $(21 \mathrm{G})$ & Passage three times through the needle & \\
\hline 27 gauge needle (27G) & Passage three times through the needle & {$[22,31]$} \\
\hline Centrifugation (Centrif.) & Centrifugation at $10,000 \times \mathrm{g}$ for $10 \mathrm{~min}$ & [21] \\
\hline $\begin{array}{l}\text { Freeze in liquid nitrogen } \\
\left(\mathrm{N}_{2}\right)\end{array}$ & Immerse liquid $\mathrm{N}_{2}$ for $2 \mathrm{~min}$ and thawed at $35^{\circ} \mathrm{C}$ for $10 \mathrm{~min}$ & [33] \\
\hline $\begin{array}{l}\text { Freezing-thawing cycles } \\
\text { (Freeze Thaw.) }\end{array}$ & $\begin{array}{l}3 \text { cycles of freezing-thawing consisting of } 15 \mathrm{~min} \text { at }-80^{\circ} \mathrm{C} \\
\text { followed by } 10 \mathrm{~min} \text { at } 35^{\circ} \mathrm{C}\end{array}$ & [18] \\
\hline \multicolumn{3}{|l|}{ Chemical } \\
\hline Triton $^{\mathrm{TM}} \mathrm{X}-100$ & $0.02 \%$ Triton $^{\mathrm{TM}} \mathrm{X}-100$ for $20 \mathrm{~min}$ at RT & [23] \\
\hline SDS & $0.5 \%$ SDS for $10 \mathrm{~min}$ at rt & [24] \\
\hline Salt treatment $(\mathrm{KCl})$ & $\begin{array}{l}\text { Centrifugation at } 10,000 \times \mathrm{g} \text { for } 10 \text { min, the supernatant was } \\
\text { replaced with } 1 \mathrm{~mL} \text { of } 0.038 \mathrm{M} \mathrm{KCl} \text {, vortexed and incubated for } 3 \mathrm{~h} \text { at RT }\end{array}$ & [19] \\
\hline
\end{tabular}


legionellae and amoebae, plates were centrifuged at $500 \times \mathrm{g}$ for $10 \mathrm{~min}$. After $2 \mathrm{~h}$ of incubation at $30^{\circ} \mathrm{C}$, the buffer was removed, and wells were washed twice with fresh and pre-warmed PAS buffer. To inactivate the remaining extracellular bacteria, co-culture suspensions were incubated for an hour at $30{ }^{\circ} \mathrm{C}$ with $50 \mathrm{ng} / \mathrm{mL}$ of gentamicin in the 1:10 PYG:PAS buffer. Then, wells were washed again, and fresh PYG:PAS buffer was added. This was denoted as time point 0 . After evaluating several incubation times (see co-culture monitoring), incubation times of $36 \mathrm{~h}$ for L. pneumophila Olda and of $24 \mathrm{~h}$ for L. pneumophila Paris were chosen.

\section{Co-culture monitoring}

Acanthamoeba co-cultures with L. pneumophila were analysed at different time points to determine the maximum possible number of $L$. pneumophila cells within an amoeba cell. This time point was considered the optimal time to apply the release methods. Briefly, $1 \mathrm{~mL}$ of the co-culture sample was washed twice by centrifugation at $1000 \times \mathrm{g}$ for $5 \mathrm{~min}$ by adding fresh phosphatebuffered saline (PBS). Then, $900 \mu \mathrm{L}$ of the supernatant was discarded, and the pellet was resuspended in the remaining PBS. From this, $10 \mu \mathrm{L}$ was placed onto a 10well Teflon slide (Medco Health Solutions, Inc., Germany). Slides were incubated at $30{ }^{\circ} \mathrm{C}$ for $30 \mathrm{~min}$ to let the cells attach to the slide surface. The samples were fixed by incubation for $10 \mathrm{~min}$ at RT in $20 \mu \mathrm{L}$ of $4 \%$ paraformaldehyde ( $\mathrm{v} / \mathrm{v}$ PBS), washing once with PBS, and dehydrating for $3 \mathrm{~min}$ in an aqueous ethanol series (50, 80 , and $96 \%)$. Cells were then stained with the MONOFLUO $^{\mathrm{ma}}$ Legionella pneumophila IFA Test Kit, which uses FITC-labelled monoclonal antibodies to detect the major outer membrane protein of L. pneumophila. Slides were then investigated using a Nikon Eclipse 8000 epifluorescence microscope, and photographs were processed with the software NIS Elements BR 2.3 (Nikon).

\section{Methods for the release of $L$. pneumophila}

Both, mechanical and chemical methods (Table 1) were first applied to the different microorganisms in pure cultures and then to the respective co-cultures. One-millilitre suspensions of L. pneumophila, Acanthamoeba and the four co-cultures were prepared as explained above. After all treatments, the suspensions were placed in an ultrasonic bath (Bandelin Sonorex, RK 100, 35 kHz, Germany) for $2 \mathrm{~min}$ to enhance release of the bacterial cells.

\section{Evaluation of the ISO 11731:1998 method for the detection of intracellular L. pneumophila}

All suspensions were processed as described in the ISO 11731:1998 for the detection and enumeration of Legionella [27]. Briefly, water samples were spiked with $10^{5}$ cells $/ \mathrm{mL}$ in $100 \mathrm{~mL}$ of distilled water. After filtering the sample through a $0.22 \mu \mathrm{m}$ polycarbonate filter, cells were recovered in $5 \mathrm{~mL}$ of Ringer solution $1 / 40$ by sonication in an ultrasound bath for $2 \mathrm{~min}$. Then, samples were divided into 3 aliquots. One aliquot was transferred to supplemented BCYE agar plates or NNA plates with $E$. coli in case of the amoebae without any further treatment. The second aliquot was transferred to the agar plates after being exposed to an acid buffer for $5 \mathrm{~min}$, and the third aliquot was incubated at $50{ }^{\circ} \mathrm{C}$ for $30 \mathrm{~min}$ before transfer to the plates [27]. For every aliquot, tenfold dilution series were made in Ringer solution 1/40 before transfer to the plates. Supplemented BCYE agar plates were incubated at $37{ }^{\circ} \mathrm{C}$ for up to 10 days and NNA plates were incubated at $30{ }^{\circ} \mathrm{C}$ for up to 7 days.

\section{Statistical analysis}

The inactivation of different microorganisms was defined as a logarithmic reduction $\left(\mathrm{N} / \mathrm{N}_{0}\right)$, whereby $\mathrm{N}_{0}$ and $\mathrm{N}$ referred to the concentration of culturable cells of $L$. pneumophila or the MPN of amoebae before and after release treatments, respectively. The results are presented (or depicted) as the mean \pm standard deviation (SD) and were plotted using GraphPad Prism 4. All data reported in this study were obtained from independent experiments performed in triplicate. The experimental conditions were statistically analysed using one-way ANOVA tests; $p$ values less than 0.05 were considered to indicate statistical significances (GraphPad Software, San Diego, CA, USA). After the ANOVA test, a Bonferroni's multiple comparison test was used to discern between the means in the case of significant differences (GraphPad Software, San Diego, CA, USA).

\section{Ethics approval and consent to participate \\ Not applicable.}

\section{Consent for publication}

Not applicable.

\section{Availability of data and materials}

Data presented in this study are complete. No supplementary files are attached.

\footnotetext{
Abbreviations

BCYE: buffered charcoal yeast extract; FITC: fluorescein isothiocyanate:

FLA: free-living amoebae; GVPC: glycine, vancomycin, polymyxin b, cycloheximide; L. pneumophila: Legionella pneumophila; MOMP: major outer membrane protein; MPN: most probable number; NNA: non-nutrient agar; PAS: amoeba page's saline; PBS: phosphate buffer; PYG: proteose-peptone-yeast extract-glucose; RT: room temperature; SD: standard deviation.
}

Competing interests

The authors declare that they have no competing interests. 


\section{Authors' contribution}

ED and SCA carried out the experimental work of the study, performed the statistical analysis, made the graph pots and drafted the manuscript. ED, SCA, RS, AKK and JW participated in the design of the study, coordination and review of the manuscript. SCA conceived the study. All authors read and approved the final manuscript.

\section{Acknowledgements}

We thank the staff of the Water Hygiene Unit, Institute for Hygiene and Applied Immunology and the Molecular Parasitology Unit, Institute of Specific Prophylaxis and Tropical Medicine, Medical University of Vienna, especially Sonja Knetsch, Andrea Lettl and Stefan Jakwerth, for their kind assistance.

\section{Funding}

This study was supported by the Austrian Science Fund (FWF, www.fwf.ac.at; Project P24535-B22) and the Austrian Federal Ministry for Science and Research (HSRSM Projekt LE103HS001).

\section{Author details}

${ }^{1}$ Institute of Specific Prophylaxis and Tropical Medicine, Department of Medical Parasitology, Medical University of Vienna, Kinderspitalgasse 15, A-1090 Vienna, Austria. ${ }^{2}$ Institute for Hygiene and Applied Immunology, Water Hygiene, Medical University of Vienna, Kinderspitalgasse 15, A-1090 Vienna, Austria. ${ }^{3}$ Interuniversity Cooperation Centre for Water \& Health, Vienna, Austria.

Received: 22 October 2015 Accepted: 14 April 2016 Published online: 26 April 2016

\section{References}

1. Marciano-Cabral F, Cabral G. Acanthamoeba spp. As agents of disease in humans. Clin Microbiol Rev. 2003;16:273-307.

2. Schuster FL, Visvesvara GS. Free-living amoebae as opportunistic and nonopportunistic pathogens of humans and animals. Int J Parasitol. 2004;34:1001-27.

3. Siddiqui R, Khan N. Biology and pathogenesis of Acanthamoeba. Parasit Vectors. 2012;5:6.

4. Barker J, Brown MRW. Review. Trojan horses of the microbial world: protozoa and the survival of bacterial pathogens in the environment. Microbiol. 1994;140:1253-9.

5. Essig A, Heinemann M, Simnacher U, Marre R. Infection of Acanthamoeba castellanii by Chlamydia pneumoniae. Appl Environ Microbiol. 1997;63(4):1396-9.

6. Greub G, Raoult D. Microorganisms resistant to free-living amoebae. Clin Microbiol Rev. 2004;17:413-33.

7. Walochnik J, Picher O, Aspöck C, Ullmann M, Aspöck H. Vergleichende untersuchungen über das verhalten von "Limax-Amöben"(acanthamoeba und hartmannella) auf kulturen verschiedener gramnegativer bakterienspezies. Mitt Österr Ges Tropenmed Parasitol. 1997:19:21-8.

8. Steinert M, Hentschel U, Hacker J. Legionella pneumophila: an aquatic microbe goes astray. FEMS Microbiol Rev. 2002;26:149-62.

9. Chaabna Z, Forey F, Reyrolle M, Jarraud S, Atlan D, Fontvieille D, Gilbert C. Molecular diversity and high virulence of Legionella pneumophila strains isolated from biofilms developed within a warm spring of a thermal spa. BMC Microbiol. 2013;13:17

10. Bartram J, Chartier Y, Lee JV, Pond K, Surman-Lee S. Legionella and the Prevention of Legionellosis. Geneva: World Health Organization; 2007.

11. Fields BS, Benson RF, Besser RE. Legionella and legionnaires' disease: 25 years of investigation. Clin Microbiol Rev. 2002;15:506-26.

12. Kim BR, Anderson JE, Mueller SA, Gaines WA, Kendall AM. Literature review: efficacy of various disinfectants against Legionella in water systems. Water Res. 2002;36:4433-44.

13. Storey MV, Winiecka-Krusnell J, Ashbolt NJ, Stenström T-A. The efficacy of heat and chlorine treatment against thermotolerant Acanthamoebae and Legionellae. Scand J Infect Dis. 2004;36:656-62.

14. Dupuy M, Mazoua S, Berne F, Bodet C, Garrec N, Herbelin P, MénardSzczebara F Oberti S, Rodier M-H, Soreau S, Wallet F, Héchard Y. Efficiency of water disinfectants against Legionella pneumophila and Acanthamoeba. Water Res. 2011:45:1087-94.

15. Cervero-Aragó S, Sommer R, Araujo RM. Effect of UV irradiation ( $253.7 \mathrm{~nm}$ ) on free Legionella and Legionella associated with its amoebae hosts. Water Res. 2014;67C:299-309.
16. Thomas V, McDonnell G, Denyer SP, Maillard J-Y. Free-living amoebae and their intracellular pathogenic microorganisms: risks for water quality. FEMS Microbiol Rev. 2010;34:231-59.

17. Robertson P, Abdelhady H, Garduño RA. The many forms of a pleomorphic bacterial pathogen the developmental network of Legionella pneumophila. Front Microbiol. 2014:5:1-20.

18. Berk SG, Ting RS, Turner GW, Ashburn RJ. Production of respirable vesicles containing live Legionella pneumophila cells by two Acanthamoeba spp. Appl Environ Microbiol. 1998;64(1):279-86.

19. Chambers R. Micrurgical studies in cell physiology: I. the action of the chlorides of $\mathrm{Na}, \mathrm{K}, \mathrm{Ca}$, and $\mathrm{Mg}$ on the protoplasm of Amoeligba proteus. J Gen Physiol. 1926:8:369-401.

20. Barker J, Scaife H, Brown MRW. Intraphagocytic growth induces an antibiotic-resistant phenotype of Legionella pneumophila. Antimicrob Agents Chemother. 1995:39:2684-8.

21. Wintermeyer E, Ludwig B, Steinert M, Schmidt B, Fischer G, Hacker J. Influence of site specifically altered Mip proteins on intracellular survival of Legionella pneumophila in eukaryotic cells. Infect Immun. 1995;63(12):4576-83.

22. Moffat JF, Tompkins LS. A quantitative model of intracellular growth of Legionella pneumophila in Acanthamoeba castellanii. Infect Immun. 1992;60:296-301.

23. Buse HY, Ashbolt NJ. Counting Legionella cells within single amoeba host cells. Appl Environ Microbiol. 2012;78:2070-2.

24. Yousuf FA, Siddiqui R, Khan NA. Acanthamoeba castellanii of the T4 genotype is a potential environmental host for Enterobacter aerogenes and Aeromonas hydrophila. Parasit Vectors. 2013;6:169.

25. Conza L, Casati S, Gaia V. Detection limits of Legionella pneumophila in environmental samples after co-culture with Acanthamoeba polyphaga. BMC Microbiol. 2013:13:49.

26. Cirillo JD, Falkow S, Tompkins LS. Growth of Legionella pneumophila in Acanthamoeba castellanii enhances invasion. Infect Immun. 1994;62:3254-61.

27. Molofsky AB, Swanson MS. Differentiate to thrive: lessons from the Legionella pneumophila life cycle. Mol Microbiol. 2004;53:29-40.

28. Schoen ME, Ashbolt NJ. An in-premise model for Legionella exposure during showering events. Water Res. 2011;45:5826-36

29. Molmeret M, Jarraud S, Mori JP, Pernin P, Forey F, Reyrolle M, Vandenesch F, Etienne J, Farge P. Different growth rates in amoeba of genotypically related environmental and clinical Legionella pneumophila strains isolated from a thermal spa. Epidemiol Infect. 2001;126:231-9.

30. Cervero-Aragó S, Rodríguez-Martínez S, Puertas-Bennasar A, Araujo RM. Effect of common drinking water disinfectants, chlorine and heat, on free Legionella and amoebae-associated Legionella. PLOS ONE. 2015;10:1-8.

31. Holden EP, Winkler HH, Wood DO, Leinbach ED. Intracellular growth of Legionella pneumophila within Acanthamoeba castellanii Neff. Infect Immun. 1984;45:18-24.

32. García MT, Jones S, Pelaz C, Millar RD, Abu Kwaik Y. Acanthamoeba polyphaga resuscitates viable non-culturable Legionella pneumophila after disinfection. Environ Microbiol. 2007:9:1267-77.

33. International Organization for Standardization: ISO 11731:1998. Water Quality - Detection and Enumeration of Legionella. 1998.

34. Lawrence C, Reyrolle M, Dubrou S, Forey F, Decludt B, Goulvestre C, MatsiotaBernard P, Etienne J, Nauciel C. Single clonal origin of a high proportion of Legionella pneumophila serogroup 1 isolates from patients and the environment in the area of Paris, France, over a 10-year period. J Clin Microbiol. 1999:37:2652-5.

35. Walochnik J, Obwaller A, Aspöck H. Correlations between morphological, molecular biological, and physiological characteristics in clinical and nonclinical isolates of Acanthamoeba spp. Appl Environ Microbiol. 2000;66:4408-13.

36. Walochnik J, Picher O, Aspöck C, Ullmann M, Sommer R, Aspöck H. Interactions of "Limax amoebae" and gram-negative bacteria: Experimental studies and review of current problems. Tokai J Exp Clin Med. 1998:23:273-8.

37. Cervero-Aragó S, Rodríguez-Martínez S, Canals O, Salvadó H, Araujo RM. Effect of thermal treatment on free-living amoeba inactivation. J Appl Microbiol. 2013;116:1-9.

38. International Organization for Standardization: ISO 8199:2005. Water Quality - General Guidance on the Enumeration of Micro-Organisms by Culture. 2005

39. Page FC. A revised classification of the Gymnamoebia (Protozoa: Sarcodina). Zool J Linnean Soc. 1976;58:61-77.

40. Declerck P, Behets J, Delaedt Y, Margineanu A, Lammertyn E, Ollevier F. Impact of non-Legionella bacteria on the uptake and intracellular replication of Legionella pneumophila in Acanthamoeba castellanii and Naegleria lovaniensis. Microb Ecol. 2005;50:536-49. 